\title{
The Effect of Probe Correctors on the Analytical Results of Non-ideal Samples
}

\author{
J. Ringnalda ${ }^{1}$, A. Genc ${ }^{1}$, L. Kovarik ${ }^{2}$ \\ ${ }^{1}$ FEI Company, 5350 NE Dawson Creek Drive, Hillsboro, OR 97124, USA \\ ${ }^{2}$ Environmental Molecular Sciences Laboratory, Pacific Northwest National Laboratory, P.O. Box \\ 999, Richland, WA 99352, USA
}

Scanning Transmission Electron Microscopy (STEM) has been enhanced in terms of resolution and probe current with the implementation of aberration correctors on the probe forming lens of a transmission electron microscope (TEM). Using the presently available techniques of measuring resolution in STEM mode, the resolution of the image, perceived to indicate the size of the electron beam (probe) is determined by measuring the extension of spatial frequencies present in the fast Fourier transform (FFT) of a STEM image. The probe aberration corrector allows a larger convergence angle to be used, thus increasing the current that can be put into a focused probe. However this probe is only really in focus in a very small crossover within the TEM sample of finite thickness (t). Realistically samples of thickness of the order of 5-15 nm benefit of probe correction because of the limited depth of field (DOF) of the aberration corrected probe at large convergence angles. Samples that are thicker either maintain the smaller probe entering into the surface through channeling, or often suffer divergence of the beam and therefore a worsening of the analytical performance as the probe travels through the sample [1,2]. For crystalline samples, because the incoming probe has more angular spread with the probe corrector, coherent diffraction effects result in more spots in the FFT leading to the perception of increased resolution, however from an analytical point of view, the probe interactions after the initial surface reaction do not enhance the spatial resolution, and this limitation has to be considered for probe corrected systems.

Before the advent of probe correction, the active area of the condenser lens was limited by the aperture size, chosen to optimize resolution and minimize beam tails. This led to use of typical convergence angles of around $10 \mathrm{mrad}$. The only way to increase the probe current for that given convergence angle, is to increase the source brightness. By utilizing a high brightness Schottky source (FEI X-FEG), about 4 times brighter than a conventional Schottky emitter, the probe current can be significantly enhanced without having to use large convergence angles or worry about decaying probe current as in a ColdFEG. For electron tomography experiments, the limited DOF of the probe causes loss of depth sensitivity in the aberration corrected STEM images and limits the resolution of 3 dimensional (3D) reconstructions especially when analyzing samples are between $50 \mathrm{~nm}$ to $100 \mathrm{~nm}$ in thickness once they are tilted [3]. To elucidate the effect of limited DOF on the analytical performance of an aberration corrected microscope in relatively thick non ideal samples, the experiments are carried out using a metallic sample with small particles (1-400 nm in size) embedded throughout the thickness of the TEM foil $(\mathrm{t}: \sim 60 \mathrm{~nm})$. The experiments were performed on the same microscope, on the same area, with the same data acquisition settings; the only variable was the semi-convergence angle $(\alpha)$ of the incident electron beam. Figure 1a is the schematic showing the effect of varying a in the extension of the focal distance $\left(d_{f}\right)$ of the probe within the TEM sample, where we expect larger $d_{f}$ and smaller variation in probe intensity by using smaller a. As seen in Figure $1 \mathrm{~b}$, there is a wide distribution of $\mathrm{Cr}$ deficient $\gamma$ particles of the Ni based superalloy shown in the $\mathrm{Cr} \mathrm{K}_{\alpha}$ elemental maps taken at varying $\alpha$ of 21 (left map) and 10 (right map) mrad, respectively. When these images are analyzed in more detail, it becomes clear that $\gamma^{\prime}$ particles that are sized below $\sim 5-10 \mathrm{~nm}$ disappear in the XEDS elemental maps taken using 
larger beam convergence of the probe with smaller DOF of $\sim 5 \mathrm{~nm}$. This result is highlighted by comparing the regions circled in red showing the location of $\gamma^{\prime}$ particles that are less than $\sim 5 \mathrm{~nm}$ width in corresponding images. To analyze this further, the spectra integrated in identical locations of the $\gamma$ particles taken at different a are compared in Figure $1 \mathrm{c}$ showing the decreased $\mathrm{Ni} / \mathrm{Cr}$ ratio of X-ray peak intensity with increased a and limited DOF as compared to extended DOF of $20 \mathrm{~nm}$ with a of 10 $\mathrm{mrad}$. The data presented in this paper shows that the analytical resolution is significantly better when a smaller convergence angle is used for the sample in this study.

\section{References}

[1] J.K. Hyun et al., Ultramicroscopy 109 (2008) 1-7.

[2] K. van Benthem et al., Ultramicroscopy 106 (2006) 1062-1068.

[3] H.L. Xin et al., Journal of Electron Microscopy (2009) 1-9.
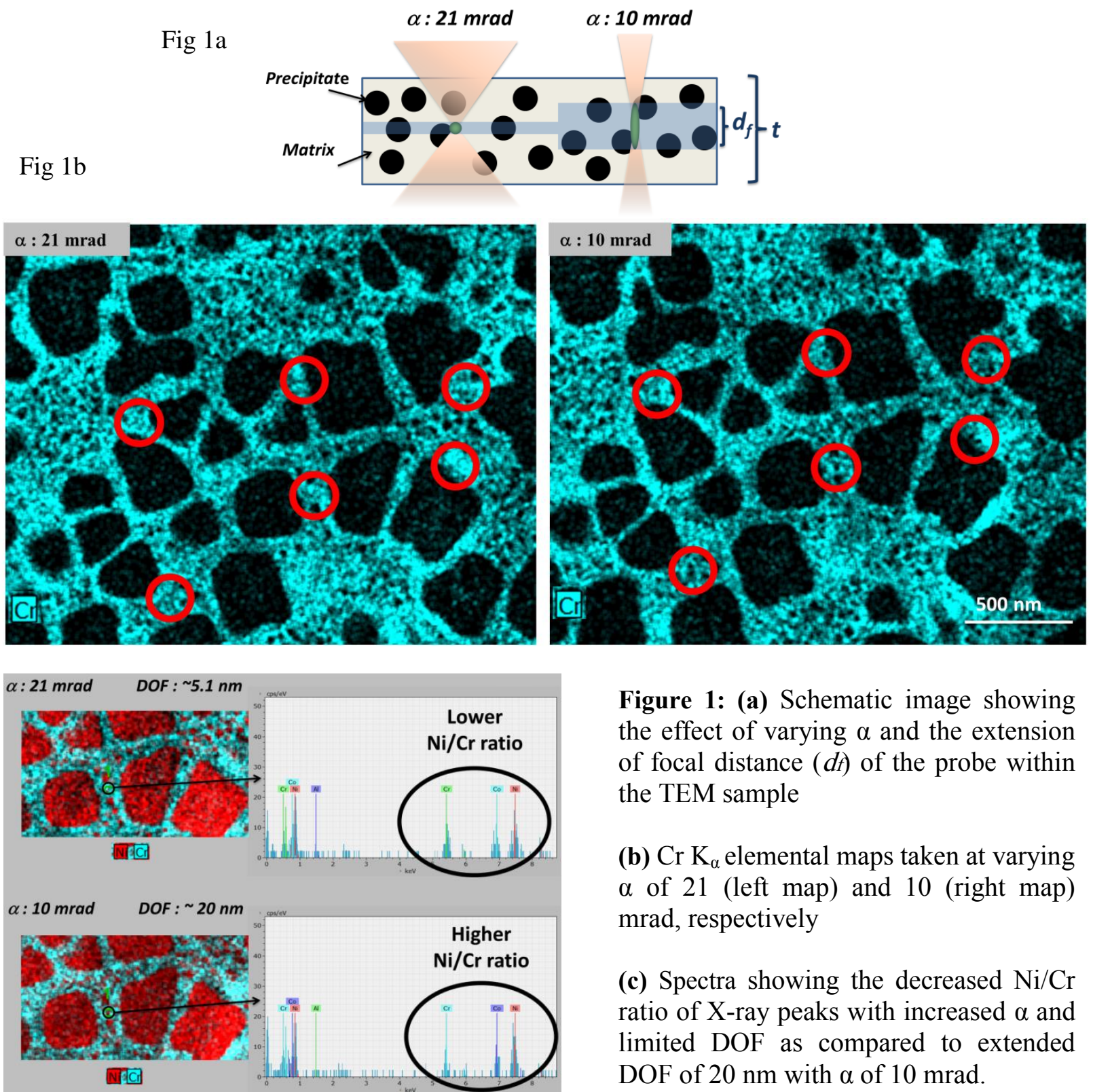

Figure 1: (a) Schematic image showing the effect of varying $\alpha$ and the extension of focal distance $(d f)$ of the probe within the TEM sample

(b) $\mathrm{Cr} \mathrm{K}_{\alpha}$ elemental maps taken at varying $\alpha$ of 21 (left map) and 10 (right map) mrad, respectively

(c) Spectra showing the decreased $\mathrm{Ni} / \mathrm{Cr}$ ratio of X-ray peaks with increased $\alpha$ and limited DOF as compared to extended DOF of $20 \mathrm{~nm}$ with $\alpha$ of $10 \mathrm{mrad}$. 